\section{The two faces of science}

Steven Yearley

Science in Action: How to Follow Scientists and Engineers Through Society. By Bruno Latour. Open University Press/Harvard University Press: 1987. Pp.274. Hbk £25, $\$ 25 ; p b k £ 9.95$.

IN COMMON with the recent generation of sociologists and historians of science, Bruno Latour is opposed to stories about the growth of science that explain beliefs in terms of their correspondence with nature. Rather, representations of how nature is emerge out of scientific disputes. All scientific and technical claims thus have a two-faced character: they come to look secure and impregnable where once they seemed provisional and contestable. Any particular representation of nature we come to accept is thus the consequence not the cause of agreement.

According to Latour, in making the next step from this position many historians and sociologists of science find themselves explaining representations of nature away. They replace the constraining influence of nature with that of "multinational firms' strategies, classes, world economic trends, national cultures ... and so on" (p.256). But, of course, the same processes that reassure scientists about their beliefs operate to re-confirm for sociologists and historians the existence of cultures and classes. Latour believes "we should be as agnostic about society as about nature" (p.256).

Latour offers an alternative: as his subtitle indicates, he wants us to follow scientists and engineers through society. Starting from the scientific paper, we follow possible challenges to the authority of science into the place of scientific work, generically termed the laboratory. Along this route we see that there are no clear boundaries between scientific and social influences. The sources of compulsion in scientific arguments are indivisibly scientific and social. Scientists require all manner of supports and resources to make their case. Again, the decision as to which of these factors are necessary and scientific ones, and which are merely accidental, can only be made with hindsight.

Instead of forlornly seeking to sort the cognitive from the social in our accounts of science, Latour suggests that we should study how scientists and technologists enlist others to their cause. The products of science (theories, designs or whatever) gain acceptance through the establishment of networks of parties who have uses for those products. Pasteur, for example, was enmeshed in a network with "health officers, veterinary surgeons and farm

there is to be said or what future studies could possibly hope to demonstrate. We cannot, for example, say that such-andsuch an organization of scientific labour increases the influence of economic considerations because we have to be agnostic about the status of such factors. The hostile reader may even feel that such seamlessness is equivalent to a sort of tautology.

Latour himself concludes by stating that his book offers "a breathing space to those who want to study independently the extensions of all [scientific and technical] networks" (p.257, my italics). Yet the main point of the book seems to be that studies and claims outside of networks are meaningless. The independent analyst is a fiction. Into whose network, then, does the book fit?

Steven Yearley is a Lecturer in the Department of Social Studies, Queen's University, Belfast $B T 71 N N, U K$

\section{Mastering models}

\section{Jeremy Sanders}

Computer-Assisted Structure Elucidation. By Neil A. B. Gray. Wiley:1986. Pp.536. $\$ 54.95, £ 52.70$.

MANY chemists enjoy the intellectual challenge of structure elucidation. To others, however, the process is merely a prelude to synthetic, mechanistic or biochemical studies, so short cuts to the structure are warmly welcomed. The most important short cuts, other than X-ray crystallography, are the spectroscopic methods: IR, NMR, UV and mass spectra all give detailed information about different aspects of molecular structure, but generally do not lead directly to a complete structure. The chemist therefore has to create a set of hypothetical structures and then test their predicted properties against the experimental evidence. The structures that fail are discarded, while those that pass are subjected to further tests. With luck, one is left with a unique correct structure. Sometimes, several candidates fit all the evidence and additional experiments are necessary; on other occasions all the structures the chemist can think of must be discarded - we tend only to propose structure types that are familiar or precedented, while nature is more creative and less prejudiced.

In this book, Neil Gray has attempted to show how computers can help in all phases of the structure-elucidation process. He explains clearly, and with abundant chemical examples, the principles behind pattern recognition, spectrum matching, and the generation and testing of structures. The book is derived from Gray's association with Djerassi's historic
DENDRAL project of the 1970s, and in places it perhaps gives undue emphasis to the details of that particular project - for example, the algorithms for generating and checking all possible structures with a particular formula are described in more detail than most chemists need. The reference lists are comprehensive, and also cover topics such as spectroscopic data bases and synthesis-analysis programs. Unfortunately the literature coverage peters out in early 1983 , so there is no mention of automated analysis (using pattern recognition algorithms) of COSY NMR spectra or of NOE data.

Gray is careful to emphasize both the strengths of the computer, as in the generation of possible structures, and its weaknesses, as in the matching of spectroscopic data to unusual structural features. We all know that the computer is indispensable for collecting and processing spectroscopic data; Gray shows us that we should also use it for generating structures as candidates in the elucidation process, because that is a mechanical job the machine does better than we do. However, it is clear that, as yet, we do not know how to program the subtle interpretation of information or the inspirational leap to the right answer, presumably because we don't know how we ourselves do it.

This, then, is an interesting book which will be useful for chemists who want to know about computers or computer scientists who want to know about chemistry. It also challenges us to ponder upon, and admire, the way that the human mind goes about the business of solving problems.

Jeremy Sanders is a Lecturer in the University Chemical Laboratory, University of Cambridge, Lensfield Road, Cambridge CB2 IEW, UK 